IJMMS 2003:8, 475-500

PII. S0161171203106230

http://ijmms.hindawi.com

(c) Hindawi Publishing Corp.

\title{
THE COMBINATORIAL STRUCTURE OF TRIGONOMETRY
}

\author{
ADEL F. ANTIPPA
}

Received 28 June 2001 and in revised form 6 January 2002

\begin{abstract}
The native mathematical language of trigonometry is combinatorial. Two interrelated combinatorial symmetric functions underlie trigonometry. We use their characteristics to derive identities for the trigonometric functions of multiple distinct angles. When applied to the sum of an infinite number of infinitesimal angles, these identities lead to the power series expansions of the trigonometric functions. When applied to the interior angles of a polygon, they lead to two general constraints satisfied by the corresponding tangents. In the case of multiple equal angles, they reduce to the Bernoulli identities. For the case of two distinct angles, they reduce to the Ptolemy identity. They can also be used to derive the De MoivreCotes identity. The above results combined provide an appropriate mathematical combinatorial language and formalism for trigonometry and more generally polygonometry. This latter is the structural language of molecular organization, and is omnipresent in the natural phenomena of molecular physics, chemistry, and biology. Polygonometry is as important in the study of moderately complex structures, as trigonometry has historically been in the study of simple structures.
\end{abstract}

2000 Mathematics Subject Classification: 51M10, 05A10, 05 E05.

1. Introduction. Trigonometry plays an important role in classical, quantum, and relativistic physics, mainly via the rotation group, Lorentz group, Fourier series, and Fourier transform. As the complexity of the physical structures studied increases, the need for the trigonometry of general polygons, or polygonometry, emerges. Polygonometry is omnipresent in the natural phenomena of molecular physics, molecular chemistry, and molecular biology [5]. It is the structural language of molecular organization. It is as important in the study of moderately complex discrete structures, as trigonometry has historically been in the study of simple structures. In solid state physics, we avoid having to deal with general polygonometry by postulating symmetry. This, in most cases, reduces the problem to one of regular polygons, which are a regular superposition of isosceles triangles. On the other hand, in molecular biology, the lack of symmetry is often an essential feature on which the proper functioning and survival of the organism depend. In such cases, postulating symmetry means postulating away the problem. We still manage to avoid general polygonometry either by approaching the problem statistically, or by doing numerical simulations. But ultimately, our profound understanding of biological structures requires a detailed analytic understanding at several length scales simultaneously, and this drives the need for polygonometry. 
This paper is organized as follows. In Section 2, we put the subject of trigonometry in perspective from the point of view of its historical origins, mathematical language, successive generalizations, hierarchy of complexity, underlying combinatorial structure, fundamental identities, and formalism. In Section 3.1, we introduce the symmetric functions $T_{m}^{n}\left(x_{1}, x_{2}, x_{3}, \ldots, x_{n}\right)$ and $U_{m}^{n}\left(x_{1}, x_{2}, x_{3}, \ldots, x_{n} ; y_{1}, y_{2}, y_{3}, \ldots, y_{n}\right)$ as well as some of their more important characteristics. These functions provide the basic mathematical language of polygonometry. In Section 3.2, we use these functions to derive polygonometric identities for the trigonometric functions of multiple distinct angles, and show that, in the special case of equal angles, they reduce to the Bernoulli identities. In Section 3.3, we make three applications of the polygonometric identities: to derive the De Moivre-Cotes identity, the power series expansions of the trigonometric functions, and two constraints on the tangents of the interior angles of a polygon. In Section 4, we show how the polygonometric identities provide a new ab initio scaling approach to trigonometry. Mathematical proofs are left to the appendices. So is the compendium of useful identities.

\section{Trigonometry in perspective}

2.1. Historical origins. Trigonometry, being intimately related to astronomy, is a very old discipline of applied mathematics that was known to the ancient Egyptian and Babylonian astronomers of the second or third millennium B.C. [1, 24]. Officially, it is usually dated back to the earliest recorded table of cords by Hipparchus of Nicaea in the second century B.C. [1, 3, 18, 23], or possibly further back to Apollonius of Perga in the third century B.C. [26, 27]. Many generalizations of traditional plane trigonometry have been developed. They range from the spherical trigonometry of Nenelaus, which goes back to the first century A.D. [3], to trigonometry on SU(3) [6], which is one among a number of relatively recent developments [9, 11, 13, 14, 20, 25].

The original language of trigonometry was geometrical, and trigonometric functions were defined in terms of the arcs and chords of a circle. Following the introduction of logarithms by John Napier in 1614 [17], the expressions for the sine and cosine functions in terms of the exponentials were discovered by Jean Bernoulli (1702), Roger Cotes (1714), Abraham De Moivre (1722), and Leonhard Euler $(1748)$ [17, 18, 24]. This established the role of the exponential function in trigonometry and opened the way to its generalization to the hyperbolic trigonometry of Vincenzo Riccati (1757), Johann H. Lambert, and William Wallace $[17,23,24]$. In this paper, we demonstrate that, beyond the exponential function, the structure of trigonometry is combinatorial and this again opens the way to more generalizations of trigonometry.

The Greek origin of the word trigonometry refers to the science of measuring ("metron") triangles ("trigonon") [18], and this has generally been the primary traditional concern of the subject [12]. Since any polygon can be decomposed into triangles, then we can consider polygonometry as a special case 
of trigonometry. On the other hand, a triangle is a special case of a polygon, and hence we can just as justifiably consider trigonometry as a special case of polygonometry. It is this second perspective that we adopt here, and it has considerable consequences for the formulation of the subject.

In as far as the number of independent angles is concerned, trigonometry can be structured hierarchically into three subtrigonometries: the trigonometry of equilateral triangles with no independent angles; the trigonometry of isosceles triangles with one independent angle; and the trigonometry of general triangles with two independent angles. The number of independent angles in each subtrigonometry defines its rank. So the trigonometry of general triangles is of rank two.

Regular planar polygons can be studied by subdividing them into isosceles triangles and hence they belong to a trigonometry of rank one. On the other hand, a general planar polygon of $p$-sides has $p-1$ independent angles, and belongs to a trigonometry of rank $p-1$, which we refer to as polygonometry. From this perspective, trigonometry becomes only the tip of the iceberg in the vast domain of polygonometry. Since, as will be demonstrated in this paper, the underlying mathematical structure of polygonometry is combinatorial, then the same is true for trigonometry. This combinatorial character of trigonometry has historically been mistaken for symmetry and is only evident in retrospect from the general perspective of polygonometry.

2.2. Combinatorial structure. The combinatorial structure of polygonometry manifests itself in the expressions for the hyperbolic trigonometric functions of multiple distinct angles. These are

$$
\begin{aligned}
\sinh \left(\theta_{1}+\theta_{2}+\cdots+\theta_{n}\right) & =\sum_{k=0}^{\lfloor(n-1) / 2\rfloor} \sum_{\substack{\ell_{1}+\ell_{2}+\cdots+\ell_{n}=2 k+1 \\
\ell_{j} \in\{0,1\}}} \prod_{j=1}^{n} \sinh ^{\ell_{j}} \theta_{j} \cosh ^{1-\ell_{j}} \theta_{j}, \\
\cosh \left(\theta_{1}+\theta_{2}+\cdots+\theta_{n}\right) & =\sum_{k=0}^{\lfloor n / 2\rfloor} \sum_{\ell_{1}+\ell_{2}+\cdots+\ell_{n}=2 k} \prod_{j=1}^{n} \sinh _{j}^{\ell_{j}} \theta_{j} \cosh ^{1-\ell_{j}} \theta_{j}, \\
\tanh \left(\theta_{1}+\theta_{2}+\cdots+\theta_{n}\right) & =\frac{\sum_{k=0}^{\lfloor(n-1) / 2\rfloor} \sum_{\ell_{1}+\ell_{2}+\cdots+\ell_{n}=2 k+1} \prod_{\ell_{j} \in\{0,1\}}^{n}}{\sum_{k=0}^{\lfloor n / 2\rfloor} \sum_{\ell_{1}+\ell_{2}+\cdots+\ell_{n}=2 k} \tanh _{\ell_{j} \in\{0,1\}} \prod_{j=1}^{n} \tanh ^{\ell_{j}} \theta_{j}}
\end{aligned}
$$

The proof of these identities is one of the main purposes of this paper. Their circular counterparts are obtained by making repeated use of the identities $\sinh i \theta=i \sin \theta, \cosh i \theta=\cos \theta$, and $\tanh i \theta=i \tan \theta$.

The above combinatorial identities set the foundations for general polygonometry. In this paper, we give a simple, but at the same time general, rigorous, and explicit derivation of them by making use of the characteristics 
of the symmetric functions $T_{m}^{n}$ and $U_{m}^{n}$, which are introduced here as the basic functions of trigonometry and polygonometry. The proof is independent of Ptolemy's and De Moivre's identities, and actually constitutes an alternative proof of these latter identities. The present approach via the symmetric functions $T_{m}^{n}$ and $U_{m}^{n}$, provides a self-contained, autonomous foundation for trigonometry.

Within the very restricted traditional scope of trigonometry, the above polygonometric identities reduce to the formulas for the sine and cosine of the sum of two angles. These latter are known since the time of Ptolemy of Alexandria [1] in the second century A.D. They were also possibly known three centuries earlier to Hipparchus of Nicaea [18]. It was not till the end of the sixteenth century that mathematics went beyond the case $n=2$, but only in the special case of multiple equal angles. This was accomplished in 1591 by François Viète who discovered the expressions for $\sin (n \theta)$ and $\cos (n \theta)$ in terms of powers of $\sin \theta$ and $\cos \theta$ up to $n=10$, using a recursive method [18, 24]. The formulas, valid for all values of $n$, for the circular functions of multiple equal angles, were found a little over a century later, in 1702, by Jakob Bernoulli $[18,24]$. Their hyperbolic counterparts were elaborated starting in the middle of the eighteenth century by Vincenzo Riccati, Johann H. Lambert, and William Wallace [17, 23, 24].

It was E. W. Hobson, towards the end of the nineteenth century, in 1891, who seems to have been the first to go beyond $n=2$ for the case of multiple distinct angles [12]. Hobson gave two proofs for the expressions of the circular functions of multiple distinct angles. The first proof, using mathematical induction, relies on Ptolemy's identity, while the second proof, using exponentials, is based on De Moivre's identity. Hobson's book [12] entitled A Treatise on Plane and Advanced Trigonometry and republished by Dover in 1957, was re-edited seven times between 1891 and 1928. In as far as advanced trigonometry is concerned, Hobson's book dominated the first third of the twentieth century.

The second third of the twentieth century was dominated by the book of Durell and Robson [8], entitled Advanced Trigonometry and re-edited 14 times in between 1930 and 1959. Durell and Robson devote one paragraph to the circular functions of multiple distinct angles. The paragraph is hidden in the section on De Moivre's theorem and its applications. Their proof is based on De Moivre's identity and is similar to the second proof of Hobson. They treat the identities for the sine and cosine functions as intermediate steps in the derivation of the identity for the tangent, and only this latter is identified with an equation number.

The last third of the twentieth century was dominated by the Advanced Trigonometry book of Miller and Walsh [19], published in 1962 and reprinted in 1977, the only English-language advanced trigonometry book still in print. Surprisingly, these authors do not mention the circular functions of multiple 
TABLE 2.1. Proofs of the polygonometric identities.

\begin{tabular}{cccccc}
\hline Year & Author & Ref. & Angles & Type & Proof \\
\hline 150 & Ptolemy & 1 & 2 & Distinct & Geometrical \\
1591 & François Viète & 18 & $\leq 10$ & Multiple & Recursive \\
1702 & Jakob Bernoulli & 24 and 18 & $n$ & Multiple & Inductive \\
1891 & E. W. Hobson & 12 & $n$ & Distinct & Inductive \\
1891 & E. W. Hobson & 12 & $n$ & Distinct & De Moivre-Cotes \\
2001 & Present work & & $n$ & Distinct & Symmetric Fuctions \\
\hline
\end{tabular}

distinct angles at all. Rather, they restrict their attention exclusively to the case of multiple equal angles. Trigonometric functions of multiple distinct angles (whether circular or hyperbolic) are also totally absent from handbooks and compendiums of mathematical formulae [2, 10, 21, 22]. A summary of the above historical sketch of the different methods of deriving polygonometric identities is given in Table 2.1.

2.3. Rosetta stone of trigonometry. Three striking facts emerge from the previous discussion concerning the identities for the trigonometric functions of multiple distinct angles: (i) their circular counterparts were discovered towards the end of the nineteenth century; (ii) in theory, this should have opened up the domain of general polygonometry, underlined the combinatorial character of trigonometry, and contributed to structural molecular biology; (iii) in practice, the circular counterparts of the polygonometric identities had an ephemeral existence, and did neither significantly integrate the cumulative mass of mathematics, nor open up the domain of polygonometry, nor bring attention to the surprising combinatorial character of trigonometry. This is a very puzzling situation. It is like discovering the Rosetta stone [18], and then losing it, without deciphering heliography.

A possible explanation may be found, at least partially, in the following observations. First, we note that the proof of Hobson is implicit. That is the reader has to fill in many steps mentally, and it is precisely in these implicit steps that the combinatorial character of the proof is hidden. Furthermore, the combinatorial character of the result was not underlined either, since Hobson did not elaborate on the notation, nor did he give any explicit symbolic definition of it. Second, Hobson was mainly concerned with triangles. According to the first sentence of his book [12], "The primary object of the science of Plane Trigonometry is to develop a method of solving triangles." Third, at the end of the nineteenth and the beginning of the twentieth century, structural molecular biology was still many decades ahead. So, it may be that the absence of an appropriate language and an appropriate formalism, in conjunction with the absence of the driving impetus of applications, may have contributed to the 
ephemeral existence of the circular counterparts of the above identities, thus, relegating to oblivion their message and potential.

\section{Polygonometry}

3.1. The symmetric functions $T_{m}^{n}$ and $U_{m}^{n}$. The symmetric functions $T_{m}^{n}\left(x_{j}\right.$; $\left.j \in N_{n}\right)$ and $U_{m}^{n}\left(x_{j}, y_{j} ; j \in N_{n}\right)$ are defined over a set of partitions according to

$$
\begin{aligned}
T_{m}^{n}\left(x_{j} ; j \in N_{n}\right) & =\sum_{\substack{\ell_{1}+\ell_{2}+\cdots+\ell_{n}=m \\
\ell_{j} \in\{0,1\}}} \prod_{j=1}^{n} x_{j}^{\ell_{j}}, \quad m \leq n, \\
U_{m}^{n}\left(x_{j}, y_{j} ; j \in N_{n}\right) & =\sum_{\substack{\ell_{1}+\ell_{2}+\cdots+\ell_{n}=m \\
\ell_{j} \in\{0,1\}}} \prod_{j=1}^{n} x_{j}^{\ell_{j}} y_{j}^{1-\ell_{j}}, \quad m \leq n,
\end{aligned}
$$

where $T_{m}^{n}\left(x_{j} ; j \in N_{n}\right)$ is the elementary symmetric function, and $U_{m}^{n}\left(x_{j}, y_{j}\right.$; $j \in N_{n}$ ) will be referred to as the associated elementary symmetric function. The sums in (3.1a) and (3.1b) are taken over all partitions of $m$ into $n$ parts $\left\{\ell_{1}, \ell_{2}, \ell_{3}, \ldots, \ell_{n}\right\}$ subject to the constraint $\ell_{j} \in\{0,1\}$. The variable $n$ is a positive integer and the variable $m$ is a nonnegative integer. $N_{n}=\{1,2, \ldots, n\}$ is, as usual, the set whose elements are the first $n$ positive integers. For $m>n$, $T_{m}^{n}=U_{m}^{n}=0$. It is easy to see that these functions are related by

$$
\begin{aligned}
& T_{m}^{n}\left(x_{1}, x_{2}, \ldots, x_{n}\right)=U_{m}^{n}\left(x_{1}, x_{2}, \ldots, x_{n} ; 1,1, \ldots, 1\right), \\
& U_{m}^{n}\left(x_{j}, y_{j} ; j \in N_{n}\right)=\left\{\prod_{j=1}^{n} y_{j}\right\} T_{m}^{n}\left(\frac{x_{j}}{y_{j}} ; j \in N_{n}\right)
\end{aligned}
$$

and that they obey the scaling laws,

$$
\begin{aligned}
T_{m}^{n}\left(a x_{j} ; j \in N_{n}\right) & \equiv a^{m} T_{m}^{n}\left(x_{j} ; j \in N_{n}\right), \\
U_{m}^{n}\left(a x_{j}, b y_{j} ; j \in N_{n}\right) & =a^{m} b^{n-m} U_{m}^{n}\left(x_{j}, y_{j} ; j \in N_{n}\right) .
\end{aligned}
$$

They also obey the recursion relations (see Appendix A),

$$
\begin{aligned}
T_{m}^{n}\left(x_{1}, x_{2}, \ldots, x_{n}\right) & =T_{m}^{n-1}\left(x_{1}, x_{2}, \ldots, x_{n-1}\right)+x_{n} T_{m-1}^{n-1}\left(x_{1}, x_{2}, \ldots, x_{n-1}\right), \\
U_{m}^{n}\left(x_{j}, y_{j} ; j \in N_{n}\right) & =y_{n} U_{m}^{n-1}\left(x_{j}, y_{j} ; j \in N_{n-1}\right)+x_{n} U_{m-1}^{n-1}\left(x_{j}, y_{j} ; j \in N_{n-1}\right),
\end{aligned}
$$

the subsidiary conditions,

$$
U_{m>n}^{n}\left(x_{j}, y_{j} ; j \in N_{n}\right)=T_{m>n}^{n}\left(x_{j} ; j \in N_{n}\right)=0,
$$


and have as limiting cases:

$$
\begin{gathered}
T_{0}^{n}\left(x_{j} ; j \in N_{n}\right)=1, \\
U_{0}^{n}\left(x_{j}, y_{j} ; j \in N_{n}\right)=\prod_{j=1}^{n} y_{j}, \\
U_{n}^{n}\left(x_{j}, y_{j} ; j \in N_{n}\right)=T_{n}^{n}\left(x_{j} ; j \in N_{n}\right)=\prod_{j=1}^{n} x_{j} .
\end{gathered}
$$

The above recursion relations and limiting cases lead to the following additional recursion relations:

$$
\begin{aligned}
& x_{n} T_{n-1}^{n-1}\left(x_{1}, x_{2}, \ldots, x_{n-1}\right)=T_{n}^{n}\left(x_{1}, x_{2}, \ldots, x_{n}\right), \\
& x_{n} U_{n-1}^{n-1}\left(x_{j}, y_{j} ; j \in N_{n-1}\right)=U_{n}^{n}\left(x_{j}, y_{j} ; j \in N_{n}\right), \\
& y_{n} U_{0}^{n-1}\left(x_{j}, y_{j} ; j \in N_{n-1}\right)=U_{0}^{n}\left(x_{j}, y_{j} ; j \in N_{n}\right) .
\end{aligned}
$$

The most important property of the $T_{m}^{n}$ and $U_{m}^{n}$ functions, as far as the present work is concerned, is that they satisfy the following "closure" relations:

$$
\begin{gathered}
\prod_{j=1}^{n}\left(x_{j}+1\right)=\sum_{m=0}^{n} T_{m}^{n}\left(x_{j} ; j \in N_{n}\right), \\
\prod_{j=1}^{n}\left(x_{j}+y_{j}\right)=\sum_{m=0}^{n} U_{m}^{n}\left(x_{j}, y_{j} ; j \in N_{n}\right)=\sum_{m=0}^{n} U_{m}^{n}\left(y_{j}, x_{j} ; j \in N_{n}\right) .
\end{gathered}
$$

The above closure relations can be easily obtained from the generating equation for the elementary symmetric function $T_{m}^{n}$ [16], combined with (3.2b) relating $T_{m}^{n}$ and $U_{m}^{n}$. Alternatively, they can be derived directly as in Appendix B. Finally, following standard combinatorial analysis [7], when all the $n$ variables are identical, the $T_{m}^{n}$ and $U_{m}^{n}$ functions collapse to

$$
\begin{gathered}
T_{m}^{n}(x, x, \ldots, x)=\left(\begin{array}{c}
n \\
m
\end{array}\right) x^{m}, \\
U_{m}^{n}(x, x, \ldots, x ; y, y, \ldots, y)=\left(\begin{array}{c}
n \\
m
\end{array}\right) x^{m} y^{n-m} .
\end{gathered}
$$

From the binomial theorem, these collapsed expressions obey the relations

$$
\begin{gathered}
\sum_{m=0}^{n} T_{m}^{n}(x, x, \ldots, x)=(1+x)^{n}, \\
\sum_{m=0}^{n} U_{m}^{n}(x, x, \ldots, x ; y, y, \ldots, y)=(x+y)^{n},
\end{gathered}
$$

which are special cases of the closure relations (3.11). Relations (3.11), as well as their special case (3.14), form the alphabet of polygonometry, and consequently of trigonometry. 
3.2. The polygonometric identities. The proof of the hyperbolic polygonometric identities (identities for the hyperbolic functions of multiple distinct angles in terms of the hyperbolic functions of the individual angles) as given by (2.1), is essentially based on the closure relations (3.11a) and (3.11b). In addition, we also need the scaling laws (3.3a) and (3.3b), and, for now at least, we use the defining relation

$$
e^{ \pm \theta}=\cosh \theta \pm \sinh \theta
$$

A scaling type definition of the trigonometric functions is introduced in Section 4 , and from it (3.15) can be derived. Note also that an inductive proof of the polygonometric identities necessarily depends on the Ptolemy identity (case of two distinct angles). The proof given here does not and, as such, provides an independent proof of Ptolemy's identity.

Appendices C (Sine functions), D (Cosine functions), E (Tangent functions), and $\mathrm{F}$ (Cotangent functions) provide a systematic grouping of polygonometric identities, which are for the most part unavailable in the literature. We believe that they will be more useful and easily applied, if they are systematically grouped together in appendices rather than introduced in the main body of the paper as they arise. Hence, we will refer to them, as needed, by their Appendix number.

\subsubsection{The sine}

THEOREM 3.1. The hyperbolic sine of multiple distinct angles is given by

$$
\sinh \left(\theta_{1}+\theta_{2}+\cdots+\theta_{n}\right)=\sum_{\substack{m=0 \\ m \text { odd }}}^{n} U_{m}^{n}\left(\sinh \theta_{j}, \cosh \theta_{j} ; j \in N_{n}\right)
$$

Proof. From the defining equation (3.15), we have

$$
\begin{aligned}
2 \sinh \left(\theta_{1}+\theta_{2}+\cdots+\theta_{n}\right) & =e^{\left(\theta_{1}+\theta_{2}+\cdots+\theta_{n}\right)}-e^{-\left(\theta_{1}+\theta_{2}+\cdots+\theta_{n}\right)}=\prod_{j=1}^{n} e^{\theta_{j}}-\prod_{j=1}^{n} e^{-\theta_{j}} \\
& =\prod_{j=1}^{n}\left(\cosh \theta_{j}+\sinh \theta_{j}\right)-\prod_{j=1}^{n}\left(\cosh \theta_{j}-\sinh \theta_{j}\right) .
\end{aligned}
$$

Due to the closure relation (3.11b), this is equivalent to

$$
\begin{aligned}
2 \sinh \left(\theta_{1}+\theta_{2}+\cdots+\theta_{n}\right)= & \sum_{m=0}^{n} U_{m}^{n}\left(\sinh \theta_{j}, \cosh \theta_{j} ; j \in N_{n}\right) \\
& -\sum_{m=0}^{n} U_{m}^{n}\left(-\sinh \theta_{j}, \cosh \theta_{j} ; j \in N_{n}\right)
\end{aligned}
$$


and making use of the scaling law (3.3b), we have

$$
\begin{aligned}
\sinh ( & \left.\theta_{1}+\theta_{2}+\cdots+\theta_{n}\right) \\
= & \sum_{m=0}^{n} \frac{1}{2}\left[1-(-1)^{m}\right] U_{m}^{n}\left(\sinh \theta_{j}, \cosh \theta_{j} ; j \in N_{n}\right),
\end{aligned}
$$

which is equivalent to (3.16). This completes the proof of the theorem.

The circular sine of multiple distinct angles can be obtained from the corresponding hyperbolic sine by making repeated use of the relations $\sinh i \theta=$ $i \sin \theta$ and $\cosh i \theta=\cos \theta$ as well as the scaling law (3.3b) to obtain (C.9) of Appendix C. When all the angles are equal, then, due to (3.13), the identities for the circular and hyperbolic sines reduce, respectively, to the Bernoulli identity (C.14) for $\sin (n \theta)[11,18]$ and to its hyperbolic counterpart (C.6) for $\sinh (n \theta)$.

3.2.2. The cosine. The hyperbolic cosine of multiple distinct angles is given by

$$
\cosh \left(\theta_{1}+\theta_{2}+\cdots+\theta_{n}\right)=\sum_{\substack{m=0 \\ m \text { even }}}^{n} U_{m}^{n}\left(\sinh \theta_{j}, \cosh \theta_{j} ; j \in N_{n}\right)
$$

The strategy of proof is identical to that used above in the case of the hyperbolic sine, and the same is true for the circular cosine, which can again be obtained from the corresponding hyperbolic cosine by making repeated use of the relations $\sinh i \theta=i \sin \theta$ and $\cosh i \theta=\cos \theta$ as well as the scaling law (3.3b) to obtain identity (D.9) of Appendix D. When all the angles are equal, then, due to (3.13), the identities for the circular and hyperbolic cosines reduce, respectively, to the Bernoulli identity (D.14) for $\cos (n \theta)[8,19]$ and to its hyperbolic counterpart (D.6) for $\cosh (n \theta)$.

3.2.3. The tangent. The hyperbolic tangent of multiple distinct angles is given by

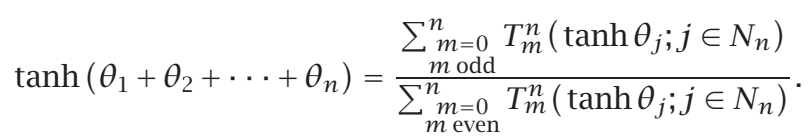

Even though this expression can be proved directly by a proof similar to that used above for the hyperbolic sine function, it is much simpler to obtain it from (3.16) and (3.20) by making use of (3.2b) relating the $T_{m}^{n}$ and $U_{m}^{n}$ functionals.

Similarly, the circular tangent of multiple distinct angles can be obtained from the above equation (3.21) by making repeated use of the identity tanh $i \theta=$ $i \tan \theta$ as well as the scaling law (3.3a). Alternatively, it can be obtained from the identities for the circular sine and circular cosine by making use, once again, 
of (3.2b) relating $T_{m}^{n}$ and $U_{m}^{n}$. The resulting identity for the circular tangent of multiple distinct angles is given by (E.5) of Appendix E. When all the angles are equal, then, due to (3.12), the identities for the circular and hyperbolic tangents reduce, respectively, to identity (E.8) for $\tan (n \theta)$ and to its hyperbolic counterpart (E.4) for $\tanh (n \theta)$.

3.2.4. The cotangent. Due to the structure of identity (3.21), as a numerator divided by a denominator, the hyperbolic cotangent of multiple distinct angles is easily obtained as

$$
\operatorname{coth}\left(\theta_{1}+\theta_{2}+\cdots+\theta_{n}\right)=\frac{\sum_{m \text { even }}^{n} T_{m}^{n}\left(\operatorname{coth}^{-1} \theta_{j} ; j \in N_{n}\right)}{\sum_{\substack{m=0 \\ m \text { odd }}}^{n} T_{m}^{n}\left(\operatorname{coth}^{-1} \theta_{j} ; j \in N_{n}\right)}
$$

Similarly, the circular cotangent of multiple distinct angles (F.5) is obtained from (E.5). Alternatively, the circular cotangent of multiple distinct angles (F.5) can be obtained from the hyperbolic cotangent (3.22) by repeated use of the identity $\operatorname{coth} i \theta=-i \cot \theta$, and the scaling law (3.3a). When all the angles are equal, then, due to (3.12), the identities for the circular and hyperbolic cotangents reduce, respectively, to identity (F.8) for $\cot (n \theta)$ and to its hyperbolic counterpart (F.4) for $\operatorname{coth}(n \theta)$. Note that the numerator and denominator of (F.4) and (F.8) have been multiplied by $\operatorname{coth}^{n} \theta$ and $\cot ^{n} \theta$, respectively.

Equations (3.16), (3.20), (3.21), and (3.22) as well as their circular counterparts and limiting cases can be written more explicitly in a number of convenient ways. A compendium is given in Appendices C, D, E, and F, respectively.

\subsection{The applications}

3.3.1. De Moivre-Cotes identity. There are a number of ways of obtaining the De Moivre-Cotes identity [18, 19] as well as its hyperbolic counterpart. The derivation presented here is based on the polygonometric identities, the binomial theorem, and the fact that the sine and cosine functions are eigenfunctions of the parity operator. Starting with (C.6) and (D.6) for the hyperbolic sine and cosine, we have

$$
\begin{aligned}
\cosh (n \theta)+\sinh (n \theta)= & \sum_{\substack{m=0 \\
m \text { even }}}^{n}\left(\begin{array}{c}
n \\
m
\end{array}\right) \sinh ^{m} \theta \cosh ^{n-m} \theta \\
& +\sum_{\substack{m=0 \\
m \text { odd }}}^{n}\left(\begin{array}{c}
n \\
m
\end{array}\right) \sinh ^{m} \theta \cosh ^{n-m} \theta \\
= & \sum_{m=0}^{n}\left(\begin{array}{c}
n \\
m
\end{array}\right) \sinh ^{m} \theta \cosh ^{n-m} \theta \\
= & (\cosh \theta+\sinh \theta)^{n} .
\end{aligned}
$$


Similarly, from (C.14) and (D.14) for the circular sine and cosine, respectively, we have

$$
\begin{aligned}
\cos (n \theta)+i \sin (n \theta)= & \sum_{\substack{m=0 \\
m \text { even }}}^{n} i^{m}\left(\begin{array}{c}
n \\
m
\end{array}\right) \sin ^{m} \theta \cos ^{n-m} \theta \\
& +i \sum_{\substack{m=0 \\
m \text { odd }}}^{n} i^{m-1}\left(\begin{array}{c}
n \\
m
\end{array}\right) \sin ^{m} \theta \cos ^{n-m} \theta \\
= & \sum_{m=0}^{n} i^{m}\left(\begin{array}{c}
n \\
m
\end{array}\right) \sin ^{m} \theta \cos ^{n-m} \theta=(\cos \theta+i \sin \theta)^{n}
\end{aligned}
$$

3.3.2. Power series expansion of the trigonometric functions. The polygonometric identities, in the special case of multiple equal angles, can be used to generate the power series of the sine and cosine functions. The procedure is the same in both cases, and so it is sufficient to make the demonstration for only one of them. For the hyperbolic sine, for example, (C.6) for $\sinh (n \theta) \operatorname{can}$ be rewritten as

$$
\sinh \theta=\sum_{\substack{m=0 \\
m \text { odd }}}^{n}\left(\begin{array}{c}
n \\
m
\end{array}\right) \sinh ^{m}\left(\frac{\theta}{n}\right) \cosh ^{n-m}\left(\frac{\theta}{n}\right) .
$$

In the limit as $n \rightarrow \infty, \sinh (\theta / n) \rightarrow \theta / n$, and $\cosh (\theta / n) \rightarrow 1$. More precisely, we have the scaling limits

$$
\lim _{n \rightarrow \infty} \sinh \left(\sinh \left(\frac{\theta}{n}\right)\right) \rightarrow \sinh \left(\frac{\theta}{n}\right), \quad \lim _{n \rightarrow \infty} \cosh \left(\sinh \left(\frac{\theta}{n}\right)\right) \longrightarrow 1 .
$$

Within the context of a self-consistent scaling type definition of the trigonometric functions, these limiting equations become the defining equations of the trigonometry. Plugging $\theta / n$ for $\sinh (\theta / n)$ and 1 for $\cosh (\theta / n)$, and letting $n \rightarrow \infty$, we obtain (this derivation can be made rigorous but we omit the details here)

$$
\sinh \theta=\lim _{n \rightarrow \infty} \sum_{\substack{m=0 \\
m \text { odd }}}^{n}\left(\begin{array}{c}
n \\
m
\end{array}\right) \frac{\theta^{m}}{n^{m}}
$$

To evaluate the limit, we use the identity

$$
\lim _{n \rightarrow \infty}\left(\begin{array}{c}
n \\
m
\end{array}\right) \frac{1}{n^{m}}=\frac{1}{m !},
$$

thus, reducing (3.27) to

$$
\sinh \theta=\sum_{\substack{m=0 \\ m \text { odd }}}^{\infty} \frac{\theta^{m}}{m !}=\sum_{k=0}^{\infty} \frac{\theta^{2 k+1}}{(2 k+1) !}
$$


which is the power series expansion or $\sinh \theta$. The derivation for $\cosh \theta$ is similar, and from the power series expansions of $\sinh \theta$ and $\cosh \theta$, we can obtain the power series of the other trigonometric functions.

3.3.3. Constraints on the interior angles of a polygon. Let the interior angles of a $p$-sided polygon be $\left\{\theta_{1}, \theta_{2}, \ldots, \theta_{p}\right\}$. The sum of these angles is given by [3]

$$
\theta_{1}+\theta_{2}+\cdots+\theta_{p}=(p-2) \pi
$$

and consequently,

$$
\begin{aligned}
& \sin \left(\theta_{1}+\theta_{2}+\cdots+\theta_{p}\right)=0, \\
& \cos \left(\theta_{1}+\theta_{2}+\cdots+\theta_{p}\right)=(-1)^{p} .
\end{aligned}
$$

Combining (C.13) of Appendix C, and (D.13) of Appendix D with the above equations (3.31), we see that the interior angles of a $p$-sided polygon obey the following relations:

$$
\begin{gathered}
\sum_{k=0}^{\lfloor(p-1) / 2\rfloor}(-1)^{k} \sum_{\substack{\ell_{1}+\ell_{2}+\cdots+\ell_{p}=2 k+1 \\
\ell_{j} \in\{0,1\}}} \prod_{j=1}^{p} \tan ^{\ell_{j}} \theta_{j}=0, \\
\sum_{k=0}^{\lfloor p / 2\rfloor}(-1)^{k+p} \sum_{\substack{\ell_{1}+\ell_{2}+\cdots+\ell_{p}=2 k \\
\ell_{j} \in\{0,1\}}}^{p} \prod_{j=1}^{p} \tan ^{\ell_{j}} \theta_{j}=\prod_{j=1}^{p} \sec \theta_{j} .
\end{gathered}
$$

For a triangle, the above equations reduce to

$$
\begin{gathered}
\sum_{j=1}^{3} \tan \theta_{j}=\prod_{j=1}^{3} \tan \theta_{j}, \\
\sum_{\substack{\ell_{1}+\ell_{2}+\ell_{3}=2 \\
\ell_{j} \in\{0,1\}}} \prod_{j=1}^{3} \tan ^{\ell_{j}} \theta_{j}=1+\prod_{j=1}^{3} \sec \theta_{j},
\end{gathered}
$$

or explicitly,

$$
\begin{aligned}
& \tan \theta_{1}+\tan \theta_{2}+\tan \theta_{3}=\tan \theta_{1} \tan \theta_{2} \tan \theta_{3}, \\
& \cot \theta_{1}+\cot \theta_{2}+\cot \theta_{3}=\cot \theta_{1} \cot \theta_{2} \cot \theta_{3}+\csc \theta_{1} \csc \theta_{2} \csc \theta_{3} .
\end{aligned}
$$

Equation (3.34) is the well-known constraint on the tangents of the angles of a triangle. Its equivalent for a quadrilateral works out to

$$
\frac{\tan \theta_{1}+\tan \theta_{2}+\tan \theta_{3}+\tan \theta_{4}}{\cot \theta_{1}+\cot \theta_{2}+\cot \theta_{3}+\cot \theta_{4}}=\tan \theta_{1} \tan \theta_{2} \tan \theta_{3} \tan \theta_{4} .
$$

Beyond the cases of the triangle and the quadrilateral, (3.32) can be worked out as needed for any value of $p$. 
4. Scaling approach to trigonometry. The derivation in Section 3.3.2 of the power series expansions for the trigonometric functions, when combined with the trigonometric identities (2.1) opens the way to a completely new approach to trigonometry. Equation (2.1) can now be looked upon as the defining equations of a whole class of sine, cosine, and tangent type functions, respectively. The defining equation being a scaling type equation, whereby the function of the whole is related (by a combinatorial expression) to the same function of its components. That is,

$$
\begin{aligned}
& f\left(\theta_{1}+\theta_{2}+\cdots+\theta_{n}\right)=\sum_{k=0}^{\lfloor(n-1) / 2\rfloor} \sum_{\substack{\ell_{1}+\ell_{2}+\cdots+\ell_{n}=2 k+1 \\
\ell_{j} \in\{0,1\}}} \prod_{j=1}^{n}\left[f\left(\theta_{j}\right)\right]^{\ell_{j}}\left[g\left(\theta_{j}\right)\right]^{1-\ell_{j}}, \\
& g\left(\theta_{1}+\theta_{2}+\cdots+\theta_{n}\right)=\sum_{k=0}^{\lfloor n / 2\rfloor} \sum_{\substack{\ell_{1}+\ell_{2}+\cdots+\ell_{n}=2 k \\
\ell_{j} \in\{0,1\}}}^{n} \prod_{j=1}^{n}\left[f\left(\theta_{j}\right)\right]^{\ell_{j}}\left[g\left(\theta_{j}\right)\right]^{1-\ell_{j}} ;
\end{aligned}
$$

the usual hyperbolic sine and cosine functions are then selected by the auxiliary conditions

$$
\lim _{\theta \rightarrow 0} \frac{f(\theta)}{\theta} \longrightarrow 1, \quad \lim _{\theta \rightarrow 0} g(\theta) \longrightarrow 1
$$

More precisely, they are selected by the scaling limits

$$
\lim _{\theta \rightarrow 0} \frac{f(f(\theta))}{f(\theta)} \longrightarrow 1, \quad \lim _{\theta \rightarrow 0} g(f(\theta)) \longrightarrow 1 .
$$

These auxiliary conditions lead, via the multiple equal angle special case of the above identities (4.1), to unique power series expansions of the trigonometric functions, thus completely defining them. Relation (3.15) between the trigonometric functions and the exponential function is then easily recovered, as well as Ptolmey's theorem which is a special case of (4.1a). We thus have a new, scaling-type combinatorial approach to trigonometry which opens the way to a new class of generalized trigonometries obtained by varying the limiting conditions appearing in (4.3).

5. Conclusion. Polygonometry finds its natural mathematical language in the symmetric functions $T_{m}^{n}$ and $U_{m}^{n}$ as defined by (3.1a) and (3.1b). These are combinatorial expressions defined over partitions. Their most important characteristics, in as far as polygonometry is concerned, are the scaling laws (3.3a) and (3.3b), and the closure relations (3.11a) and (3.11b). These functions provide a simple expression as well as a simple, explicit, and elegant derivation of the Polygonometric identities (trigonometric functions of multiple distinct angles in terms of the trigonometric functions of the individual angles). These 
latter identities, in turn, provide a complete self-coherent structure for general polygonometry, permitting among other things the derivation of the Ptolemy identity, the De Moivre-Cotes identity, the Bernoulli identities, and power series expansions for the trigonometric functions. They also provide the tools to handle general polygons, which are expected to play an important role in detailed analytic structural molecular modeling, and in moderately complex discrete structures in general.

\section{APPENDICES}

\section{A. Recursion relations}

THEOREM A.1. The symmetric functions $U_{m}^{n}\left(x_{j}, y_{j} ; j \in N_{n}\right)$ and $T_{m}^{n}\left(x_{j} ; j \in\right.$ $N_{n}$ ) obey the recursion relations

$$
\begin{gathered}
U_{m}^{n}\left(x_{j}, y_{j} ; j \in N_{n}\right)=y_{n} U_{m}^{n-1}\left(x_{j}, y_{j} ; j \in N_{n-1}\right)+x_{n} U_{m-1}^{n-1}\left(x_{j}, y_{j} ; j \in N_{n-1}\right) \\
T_{m}^{n}\left(x_{j} ; j \in N_{n}\right)=T_{m}^{n-1}\left(x_{j} ; j \in N_{n-1}\right)+x_{n} T_{m-1}^{n-1}\left(x_{j} ; j \in N_{n-1}\right) .
\end{gathered}
$$

Proof. The functions $U_{m}^{n}\left(x_{j}, y_{j} ; j \in N_{n}\right)$ are defined by

$$
U_{m}^{n}\left(x_{j}, y_{j} ; j \in N_{n}\right)=\sum_{\substack{\ell_{1}+\ell_{2}+\cdots+\ell_{n}=m \\ \ell_{j} \in(0,1)}} \prod_{j=1}^{n} x_{j}^{\ell_{j}} y_{j}^{1-\ell_{j}}
$$

Since $\ell_{n} \in\{0,1\}$, then $\ell_{n}$ can either take the value 0 or the value 1 . Thus, the above sum can be separated into two parts, one in which $\ell_{n}$ is held fixed at the value 0 and the other in which $\ell_{n}$ is held fixed at the value 1 :

$$
\begin{aligned}
U_{m}^{n}\left(x_{j}, y_{j} ; j \in N_{n}\right)= & \sum_{\substack{\ell_{1}+\ell_{2}+\cdots+\ell_{n}=m \\
\ell_{j} \in(0,1) \text { for } j \neq n \\
\ell_{n}=0}} \prod_{j=1}^{n} x_{j}^{\ell_{j}} y_{j}^{1-\ell_{j}} \\
& +\sum_{\substack{\ell_{1}+\ell_{2}+\cdots+\ell_{n}=m \\
\ell_{j} \in(0,1) \text { for } j \neq n \\
\ell_{n}=1}}^{n} \prod_{j=1}^{n} x_{j}^{\ell_{j}} y_{j}^{1-\ell_{j}} .
\end{aligned}
$$

Applying the respective constraints on $\ell_{n}$ in each sum, we obtain

$$
\begin{aligned}
U_{m}^{n}\left(x_{j}, y_{j} ; j \in N_{n}\right)= & y_{n} \sum_{\substack{\ell_{1}+\ell_{2}+\cdots+\ell_{n-1}=m \\
\ell_{j} \in(0,1)}} \prod_{j=1}^{n-1} x_{j}^{\ell_{j}} y_{j}^{1-\ell_{j}} \\
& +x_{n} \sum_{\substack{\ell_{1}+\ell_{2}+\cdots+\ell_{n-1}=m-1 \\
\ell_{j} \in(0,1)}} \prod_{j=1}^{n-1} x_{j}^{\ell_{j}} y_{j}^{1-\ell_{j}}
\end{aligned}
$$


which, due to definition (A.3), is equivalent to the recursion relation

$$
\begin{aligned}
U_{m}^{n}\left(x_{j}, y_{j} ; j \in N_{n}\right)= & y_{n} U_{m}^{n-1}\left(x_{j}, y_{j} ; j \in N_{n-1}\right) \\
& +x_{n} U_{m-1}^{n-1}\left(x_{j}, y_{j} ; j \in N_{n-1}\right)
\end{aligned}
$$

This proves the recursion relation (A.1). The recursion relation (A.2) for $T_{m}^{n}$ then follows by setting, in (A.6), $y_{j}=1$ for all $j \in N_{n}$ and making use of (3.2a).

B. Closure relations. We will first prove the closure relation (3.11b) for $U_{m}^{n}$. The closure relation (3.11a) for $T_{m}^{n}$ then follows in a straightforward way due to $(3.2 \mathrm{a})$.

THEOREM B.1. The symmetric function $U_{m}^{n}\left(x_{j}, y_{j} ; j \in N_{n}\right)$ obeys the closure relation:

$$
\prod_{j=1}^{n}\left(x_{j}+y_{j}\right)=\sum_{m=0}^{n} U_{m}^{n}\left(x_{j}, y_{j} ; j \in N_{n}\right)
$$

PROOF. The proof is carried out by mathematical induction on $n$. First, for $n=1$, (B.1) reduces to

$$
\left(x_{1}+y_{1}\right)=U_{0}^{1}\left(x_{1}, y_{1}\right)+U_{1}^{1}\left(x_{1}, y_{1}\right)=x_{1}+y_{1}
$$

where the last equality follows from (3.7b) and (3.8). Hence (B.1) is valid for $n=1$. Next, we assume the equation to be valid for $n-1$ and prove its validity for $n$. We have

$$
\begin{aligned}
\prod_{i=1}^{n}\left(x_{i}+y_{i}\right) & =\left[\prod_{i=1}^{n-1}\left(x_{i}+y_{i}\right)\right]\left(x_{n}+y_{n}\right) \\
& =\left[\sum_{m=0}^{n-1} U_{m}^{n-1}\left(x_{j}, y_{j} ; j \in N_{n-1}\right)\right]\left(x_{n}+y_{n}\right)
\end{aligned}
$$

where the second equality follows from the induction hypothesis. To produce a $U_{m}^{n}$ term from the $U_{m}^{n-1}$ term appearing in (B.3) we only have at our disposal the recursion relations (3.5), (3.9b). Thus we manipulate the above expression (B.3) in order to produce summands that are in the form of the right-hand side of these recursion relations. Starting with the result of (B.3), we perform the 
following manipulations:

$$
\begin{aligned}
\prod_{i=1}^{n}\left(x_{i}+y_{i}\right)= & \sum_{m=0}^{n-1} x_{n} U_{m}^{n-1}\left(x_{j}, y_{j} ; j \in N_{n-1}\right)+\sum_{m=0}^{n-1} y_{n} U_{m}^{n-1}\left(x_{j}, y_{j} ; j \in N_{n-1}\right) \\
= & \sum_{m=1}^{n} x_{n} U_{m-1}^{n-1}\left(x_{j}, y_{j} ; j \in N_{n-1}\right)+\sum_{m=0}^{n-1} y_{n} U_{m}^{n-1}\left(x_{j}, y_{j} ; j \in N_{n-1}\right) \\
= & \sum_{m=1}^{n-1}\left[x_{n} U_{m-1}^{n-1}\left(x_{j}, y_{j} ; j \in N_{n-1}\right)+y_{n} U_{m}^{n-1}\left(x_{j}, y_{j} ; j \in N_{n-1}\right)\right] \\
& +x_{n} U_{n-1}^{n-1}\left(x_{j}, y_{j} ; j \in N_{n-1}\right)+y_{n} U_{0}^{n-1}\left(x_{j}, y_{j} ; j \in N_{n-1}\right),
\end{aligned}
$$

and due to the recursion relations (3.5) and (3.9b), the above expression reduces to

$$
\begin{aligned}
\prod_{i=1}^{n}\left(x_{i}+y_{i}\right)= & \sum_{m=1}^{n-1} U_{m}^{n}\left(x_{j}, y_{j} ; j \in N_{n}\right) \\
& +U_{n}^{n}\left(x_{j}, y_{j} ; j \in N_{n}\right)+U_{0}^{n}\left(x_{j}, y_{j} ; j \in N_{n}\right),
\end{aligned}
$$

which is equivalent to (B.1). Hence the proof by induction is completed and (B.1) is true for all positive integer values of $n$.

\section{Identities for sine functions}

HyPERbolic SINE. The hyperbolic sine of multiple distinct angles is given by

$$
\begin{aligned}
\sinh \left(\theta_{1}+\theta_{2}+\cdots+\theta_{n}\right) & =\sum_{\substack{m=0 \\
m \text { odd }}}^{n} U_{m}^{n}\left(\sinh \theta_{j}, \cosh \theta_{j} ; j \in N_{n}\right), \\
\sinh \left(\theta_{1}+\theta_{2}+\cdots+\theta_{n}\right) & =\sum_{k=0}^{\lfloor(n-1) / 2\rfloor} U_{2 k+1}^{n}\left(\sinh \theta_{j}, \cosh \theta_{j} ; j \in N_{n}\right) .
\end{aligned}
$$

Due to relation (3.2a) between $T_{m}^{n}$ and $U_{m}^{n}$, the above equations are equivalent to

$$
\begin{aligned}
\frac{\sinh \left(\theta_{1}+\theta_{2}+\cdots+\theta_{n}\right)}{\prod_{j=1}^{n} \cosh \theta_{j}} & =\sum_{\substack{m=0 \\
m \text { odd } \\
\lfloor(n-1) / 2\rfloor}}^{n} T_{m}^{n}\left(\tanh \theta_{j} ; j \in N_{n}\right) \\
& =\sum_{k=0} T_{2 k+1}^{n}\left(\tanh \theta_{j} ; j \in N_{n}\right)
\end{aligned}
$$


and using definitions (3.1) for $T_{m}^{n}$ and $U_{m}^{n}$, (C.2) and (C.3) can be rewritten explicitly as

$$
\begin{aligned}
& \sinh \left(\theta_{1}+\theta_{2}+\cdots+\theta_{n}\right)=\sum_{k=0}^{\lfloor(n-1) / 2\rfloor} \sum_{\substack{\ell_{1}+\ell_{2}+\cdots+\ell_{n}=2 k+1 \\
\ell_{j} \in\{0,1\}}} \prod_{j=1}^{n} \sinh ^{\ell_{j}} \theta_{j} \cosh ^{1-\ell_{j}} \theta_{j}, \\
& \frac{\sinh \left(\theta_{1}+\theta_{2}+\cdots+\theta_{n}\right)}{\prod_{j=1}^{n} \cosh \theta_{j}}=\sum_{k=0}^{\lfloor(n-1) / 2\rfloor} \sum_{\substack{\ell_{1}+\ell_{2}+\cdots+\ell_{n}=2 k+1 \\
\ell_{j} \in\{0,1\}}}^{n} \prod_{j=1}^{n} \tanh ^{\ell_{j}} \theta_{j} .
\end{aligned}
$$

When all the angles are equal, the above expressions reduce to

$$
\begin{aligned}
& \sinh (n \theta)=\sum_{\substack{m=0 \\
m \text { odd }}}^{n}\left(\begin{array}{c}
n \\
m
\end{array}\right) \sinh ^{m} \theta \cosh ^{n-m} \theta, \\
& \sinh (n \theta)=\sum_{k=0}^{\lfloor(n-1) / 2\rfloor}\left(\begin{array}{c}
n \\
2 k+1
\end{array}\right) \sinh ^{2 k+1} \theta \cosh ^{n-2 k-1} \theta, \\
& \frac{\sinh (n \theta)}{\cosh ^{n} \theta}=\sum_{\substack{m=0 \\
m \text { odd }}}^{n}\left(\begin{array}{c}
n \\
m
\end{array}\right) \tanh ^{m} \theta=\sum_{k=0}^{\lfloor(n-1) / 2\rfloor}\left(\begin{array}{c}
n \\
2 k+1
\end{array}\right) \tanh ^{2 k+1} \theta .
\end{aligned}
$$

Circular Sine. The circular sine of multiple distinct angles is given by

$$
\begin{aligned}
\sin \left(\theta_{1}+\theta_{2}+\cdots+\theta_{n}\right) & =\sum_{\substack{m=0 \\
m \text { odd }}}^{n} i^{m-1} U_{m}^{n}\left(\sin \theta_{j}, \cos \theta_{j} ; j \in N_{n}\right) \\
\sin \left(\theta_{1}+\theta_{2}+\cdots+\theta_{n}\right) & =\sum_{k=0}^{\lfloor(n-1) / 2\rfloor}(-1)^{k} U_{2 k+1}^{n}\left(\sin \theta_{j}, \cos \theta_{j} ; j \in N_{n}\right) .
\end{aligned}
$$

Due to relation (3.2a) between $T_{m}^{n}$ and $U_{m}^{n}$, the above equations are equivalent to

$$
\begin{aligned}
\frac{\sin \left(\theta_{1}+\theta_{2}+\cdots+\theta_{n}\right)}{\prod_{j=1}^{n} \cos \theta_{j}} & =\sum_{\substack{m=0 \\
m \text { odd }}}^{n} i^{m-1} T_{m}^{n}\left(\tan \theta_{j} ; j \in N_{n}\right) \\
& =\sum_{k=0}^{\lfloor(n-1) / 2\rfloor}(-1)^{k} T_{2 k+1}^{n}\left(\tan \theta_{j} ; j \in N_{n}\right)
\end{aligned}
$$


and using definitions (3.1) for $T_{m}^{n}$ and $U_{m}^{n}$, (C.10) and (C.11) are rewritten explicitly as

$$
\begin{aligned}
& \sin \left(\theta_{1}+\theta_{2}+\cdots+\theta_{n}\right)=\sum_{k=0}^{\lfloor(n-1) / 2\rfloor}(-1)^{k} \sum_{\substack{\ell_{1}+\ell_{2}+\cdots+\ell_{n}=2 k+1 \\
\ell_{j} \in\{0,1\}}} \prod_{j=1}^{n} \sin ^{\ell_{j}} \theta_{j} \cos ^{1-\ell_{j}} \theta_{j}, \\
& \frac{\sin \left(\theta_{1}+\theta_{2}+\cdots+\theta_{n}\right)}{\prod_{j=1}^{n} \cos \theta_{j}}=\sum_{k=0}^{\lfloor(n-1) / 2\rfloor}(-1)^{k} \sum_{\substack{\ell_{1}+\ell_{2}+\cdots+\ell_{n}=2 k+1 \\
\ell_{j} \in\{0,1\}}}^{n} \prod_{j=1}^{n} \tan ^{\ell_{j}} \theta_{j} .
\end{aligned}
$$

When all the angles are equal, the above expressions reduce to

$$
\begin{aligned}
& \sin (n \theta)=\sum_{\substack{m=0 \\
m \text { odd }}}^{n} i^{m-1}\left(\begin{array}{c}
n \\
m
\end{array}\right) \sin ^{m} \theta \cos ^{n-m} \theta, \\
& \sin (n \theta)=\sum_{k=0}^{\lfloor(n-1) / 2\rfloor}(-1)^{k}\left(\begin{array}{c}
n \\
2 k+1
\end{array}\right) \sin ^{2 k+1} \theta \cos ^{n-2 k-1} \theta, \\
& \frac{\sin (n \theta)}{\cos ^{n} \theta}=\sum_{\substack{m=0 \\
m \text { odd }}}^{n} i^{m-1}\left(\begin{array}{c}
n \\
m
\end{array}\right) \tan ^{m} \theta=\sum_{k=0}^{\lfloor(n-1) / 2\rfloor}(-1)^{k}\left(\begin{array}{c}
n \\
2 k+1
\end{array}\right) \tan ^{2 k+1} \theta .
\end{aligned}
$$

\section{Identities for cosine functions}

Hyperbolic cosine. The hyperbolic cosine of multiple distinct angles is given by

$$
\begin{aligned}
\cosh \left(\theta_{1}+\theta_{2}+\cdots+\theta_{n}\right) & =\sum_{\substack{m=0 \\
m \text { even }}}^{n} U_{m}^{n}\left(\sinh \theta_{j}, \cosh \theta_{j} ; j \in N_{n}\right), \\
\cosh \left(\theta_{1}+\theta_{2}+\cdots+\theta_{n}\right) & =\sum_{k=0}^{\lfloor n / 2\rfloor} U_{2 k}^{n}\left(\sinh \theta_{j}, \cosh \theta_{j} ; j \in N_{n}\right) .
\end{aligned}
$$

Due to relation (3.2a) between $T_{m}^{n}$ and $U_{m}^{n}$, the above equations are equivalent to

$$
\begin{aligned}
\frac{\cosh \left(\theta_{1}+\theta_{2}+\cdots+\theta_{n}\right)}{\prod_{j=1}^{n} \cosh \theta_{j}} & =\sum_{\substack{m=0 \\
m \text { even }}}^{n} T_{m}^{n}\left(\tanh \theta_{j} ; j \in N_{n}\right) \\
& =\sum_{k=0}^{\lfloor n / 2\rfloor} T_{2 k}^{n}\left(\tanh \theta_{j} ; j \in N_{n}\right),
\end{aligned}
$$


and using definitions (3.1) for $T_{m}^{n}$ and $U_{m}^{n}$, (D.2) and (D.3) are rewritten explicitly as

$$
\begin{aligned}
& \cosh \left(\theta_{1}+\theta_{2}+\cdots+\theta_{n}\right)=\sum_{k=0}^{\lfloor n / 2\rfloor} \sum_{\substack{\ell_{1}+\ell_{2}+\cdots+\ell_{n}=2 k \\
\ell_{j} \in\{0,1\}}} \prod_{j=1}^{n} \sinh ^{\ell_{j}} \theta_{j} \cosh ^{1-\ell_{j}} \theta_{j}, \\
& \frac{\cosh \left(\theta_{1}+\theta_{2}+\cdots+\theta_{n}\right)}{\prod_{j=1}^{n} \cosh \theta_{j}}=\sum_{k=0}^{\lfloor n / 2\rfloor} \sum_{\substack{\ell_{1}+\ell_{2}+\cdots+\ell_{n}=2 k \\
\ell_{j} \in\{0,1\}}} \prod_{j=1}^{n} \tanh ^{\ell_{j}} \theta_{j} .
\end{aligned}
$$

When all the angles are equal, the above expressions reduce to

$$
\begin{aligned}
& \cosh (n \theta)=\sum_{\substack{m=0 \\
m \text { even }}}^{n}\left(\begin{array}{c}
n \\
m
\end{array}\right) \sinh ^{m} \theta \cosh ^{n-m} \theta, \\
& \cosh (n \theta)=\sum_{k=0}^{\lfloor n / 2\rfloor}\left(\begin{array}{c}
n \\
2 k
\end{array}\right) \sinh ^{2 k} \theta \cosh ^{n-2 k} \theta, \\
& \frac{\cosh (n \theta)}{\cosh ^{n} \theta}=\sum_{\substack{m=0 \\
m \text { even }}}^{n}\left(\begin{array}{c}
n \\
m
\end{array}\right) \tanh ^{m} \theta=\sum_{k=0}^{\lfloor n / 2\rfloor}\left(\begin{array}{c}
n \\
2 k
\end{array}\right) \tanh ^{2 k} \theta .
\end{aligned}
$$

Circular cosine. The circular cosine of multiple distinct angles is given by

$$
\begin{aligned}
& \cos \left(\theta_{1}+\theta_{2}+\cdots+\theta_{n}\right)=\sum_{\substack{m=0 \\
m \text { even }}}^{n} i^{m} U_{m}^{n}\left(\sin \theta_{j}, \cos \theta_{j} ; j \in N_{n}\right), \\
& \cos \left(\theta_{1}+\theta_{2}+\cdots+\theta_{n}\right)=\sum_{k=0}^{\lfloor n / 2\rfloor}(-1)^{k} U_{2 k}^{n}\left(\sin \theta_{j}, \cos \theta_{j} ; j \in N_{n}\right) .
\end{aligned}
$$

Due to relation (3.2a) between $T_{m}^{n}$ and $U_{m}^{n}$, the above equations are equivalent to

$$
\begin{aligned}
\frac{\cos \left(\theta_{1}+\theta_{2}+\cdots+\theta_{n}\right)}{\prod_{j=1}^{n} \cos \theta_{j}} & =\sum_{\substack{m=0 \\
m \text { even }}}^{n} i^{m} T_{m}^{n}\left(\tan \theta_{j} ; j \in N_{n}\right) \\
& =\sum_{k=0}^{\lfloor n / 2\rfloor}(-1)^{k} T_{2 k}^{n}\left(\tan \theta_{j} ; j \in N_{n}\right),
\end{aligned}
$$


and using definitions (3.1) for $T_{m}^{n}$ and $U_{m}^{n}$, (D.10) and (D.11) are rewritten explicitly as

$$
\begin{aligned}
& \cos \left(\theta_{1}+\theta_{2}+\cdots+\theta_{n}\right)=\sum_{k=0}^{\lfloor n / 2\rfloor}(-1)^{k} \sum_{\substack{\ell_{1}+\ell_{2}+\cdots+\ell_{n}=2 k \\
\ell_{j} \in\{0,1\}}} \prod_{j=1}^{n} \sin ^{\ell_{j}} \theta_{j} \cos ^{1-\ell_{j}} \theta_{j}, \\
& \frac{\cos \left(\theta_{1}+\theta_{2}+\cdots+\theta_{n}\right)}{\prod_{j=1}^{n} \cos \theta_{j}}=\sum_{k=0}^{\lfloor n / 2\rfloor}(-1)^{k} \sum_{\substack{\ell_{1}+\ell_{2}+\cdots+\ell_{n}=2 k \\
\ell_{j} \in\{0,1\}}} \prod_{j=1}^{n} \tan ^{\ell_{j}} \theta_{j}
\end{aligned}
$$

When all the angles are equal, the above expressions reduce to

$$
\begin{aligned}
& \cos (n \theta)=\sum_{\substack{m=0 \\
m \text { even }}}^{n} i^{m}\left(\begin{array}{c}
n \\
m
\end{array}\right) \sin ^{m} \theta \cos ^{n-m} \theta, \\
& \cos (n \theta)=\sum_{k=0}^{\lfloor n / 2\rfloor}(-1)^{k}\left(\begin{array}{c}
n \\
2 k
\end{array}\right) \sin ^{2 k} \theta \cos ^{n-2 k} \theta, \\
& \frac{\cos (n \theta)}{\cos ^{n} \theta}=\sum_{\substack{m=0 \\
m \text { even }}}^{n} i^{m}\left(\begin{array}{c}
n \\
m
\end{array}\right) \tan ^{m} \theta=\sum_{k=0}^{\lfloor n / 2\rfloor}(-1)^{k}\left(\begin{array}{c}
n \\
2 k
\end{array}\right) \tan ^{2 k} \theta .
\end{aligned}
$$

\section{E. Identities for tangent functions}

HyPERBolic TANGENT. The hyperbolic tangent of multiple distinct angles is given by

$$
\begin{aligned}
\tanh \left(\theta_{1}+\theta_{2}+\cdots+\theta_{n}\right) & =\frac{\sum_{m=0}^{n} T_{m \text { odd }}}{\sum_{m}^{n}\left(\operatorname{manh} \theta_{j} ; j \in N_{n}\right)} T_{m}^{n}\left(\tanh \theta_{j} ; j \in N_{n}\right) \\
\tanh \left(\theta_{1}+\theta_{2}+\cdots+\theta_{n}\right) & =\frac{\sum_{k=0}^{\lfloor(n-1) / 2\rfloor} T_{2 k+1}^{n}\left(\tanh \theta_{j} ; j \in N_{n}\right)}{\sum_{k=0}^{\lfloor n / 2\rfloor} T_{2 k}^{n}\left(\tanh \theta_{j} ; j \in N_{n}\right)},
\end{aligned}
$$

and using definition (3.1a) for $T_{m}^{n}$, this is rewritten explicitly as

$$
\tanh \left(\theta_{1}+\theta_{2}+\cdots+\theta_{n}\right)=\frac{\sum_{k=0}^{\lfloor(n-1) / 2\rfloor} \sum_{\ell_{1}+\ell_{2}+\cdots+\ell_{n}=2 k+1} \prod_{j=1}^{n} \tanh _{\ell_{j} \in\{0,1\}} \operatorname{lan}_{j} \theta_{j}}{\sum_{k=0}^{\lfloor n / 2\rfloor} \sum_{\ell_{1}+\ell_{2}+\cdots+\ell_{n}=2 k} \prod_{j=1}^{n} \tanh ^{\ell_{j} \in\{0,1\}} \theta_{j}}
$$


When all the angles are equal, the above expressions reduce to

$$
\tanh (n \theta)=\frac{\sum_{m=0}^{n}\left(\begin{array}{c}
n \\
m
\end{array}\right) \tanh ^{m} \theta}{\sum_{\substack{m=0 \\
m \text { even }}}^{n}\left(\begin{array}{c}
n \\
m
\end{array}\right) \tanh ^{m} \theta}=\frac{\sum_{k=0}^{\lfloor(n-1) / 2\rfloor}\left(\begin{array}{c}
n \\
2 k+1
\end{array}\right) \tanh ^{2 k+1} \theta}{\sum_{k=0}^{\lfloor n / 2\rfloor}\left(\begin{array}{c}
n \\
2 k
\end{array}\right) \tanh ^{2 k} \theta}
$$

Circular TANGent. The circular tangent of multiple distinct angles is given by

$$
\begin{aligned}
\tan \left(\theta_{1}+\theta_{2}+\cdots+\theta_{n}\right) & =\frac{\sum_{m \text { odd }}^{n} i^{m-1} T_{m}^{n}\left(\tan \theta_{j} ; j \in N_{n}\right)}{\sum_{m=0}^{n} i_{m}^{m} T_{m}^{n}\left(\tan \theta_{j} ; j \in N_{n}\right)}, \\
\tan \left(\theta_{1}+\theta_{2}+\cdots+\theta_{n}\right) & =\frac{\sum_{k=0}^{\lfloor(n-1) / 2\rfloor}(-1)^{k} T_{2 k+1}^{n}\left(\tan \theta_{j} ; j=1,2, \ldots, n\right)}{\sum_{k=0}^{\lfloor n / 2\rfloor}(-1)^{k} T_{2 k}^{n}\left(\tan \theta_{j} ; j=1,2, \ldots, n\right)},
\end{aligned}
$$

and using definition (3.1a) for $T_{m}^{n}$, this is rewritten explicitly as

$$
\tan \left(\theta_{1}+\theta_{2}+\cdots+\theta_{n}\right)=\frac{\sum_{k=0}^{\lfloor(n-1) / 2\rfloor}(-1)^{k} \sum_{\ell_{1}+\ell_{2}+\cdots+\ell_{n}=2 k+1} \prod_{j=1}^{n} \tan ^{\ell_{j} \in\{0,1\}} \theta_{j}}{\sum_{k=0}^{\lfloor n / 2\rfloor}(-1)^{k} \sum_{\ell_{1}+\ell_{2}+\cdots+\ell_{n}=2 k} \prod_{j=1}^{n} \tan ^{\ell_{j} \in\{0,1\}} \theta_{j}}
$$

When all the angles are equal, the above expressions reduce to

$$
\tan (n \theta)=\frac{\sum_{m=0}^{n} i_{m \text { odd }}^{m-1}\left(\begin{array}{c}
n \\
m
\end{array}\right) \tan ^{m} \theta}{\sum_{\substack{m=0 \\
m \text { even }}}^{n} i^{m}\left(\begin{array}{c}
n \\
m
\end{array}\right) \tan ^{m} \theta}=\frac{\sum_{k=0}^{\lfloor(n-1) / 2\rfloor}(-1)^{k}\left(\begin{array}{c}
n \\
2 k+1
\end{array}\right) \tan ^{2 k+1} \theta}{\sum_{k=0}^{\lfloor n / 2\rfloor}(-1)^{k}\left(\begin{array}{c}
n \\
2 k
\end{array}\right) \tan ^{2 k} \theta} .
$$

\section{F. Identities for cotangent functions}

HyPERBolic COTANGENT. The hyperbolic cotangent of multiple distinct angles is given by

$$
\begin{aligned}
\operatorname{coth}\left(\theta_{1}+\theta_{2}+\cdots+\theta_{n}\right) & =\frac{\sum_{m \text { even }}^{n} T_{m}^{n}\left(\operatorname{coth}^{-1} \theta_{j} ; j \in N_{n}\right)}{\sum_{m=0}^{n} T_{m}^{n}\left(\operatorname{coth}^{-1} \theta_{j} ; j \in N_{n}\right)}, \\
\operatorname{coth}\left(\theta_{1}+\theta_{2}+\cdots+\theta_{n}\right) & =\frac{\sum_{k=0}^{\lfloor n / 2\rfloor} T_{2 k}^{n}\left(\operatorname{coth}^{-1} \theta_{j} ; j=1,2, \ldots, n\right)}{\sum_{k=0}^{\lfloor(n-1) / 2\rfloor} T_{2 k+1}^{n}\left(\operatorname{coth}^{-1} \theta_{j} ; j=1,2, \ldots, n\right)},
\end{aligned}
$$


and using definition (3.1a) for $T_{m}^{n}$, this is rewritten explicitly as

$$
\operatorname{coth}\left(\theta_{1}+\theta_{2}+\cdots+\theta_{n}\right)=\frac{\sum_{k=0}^{\lfloor n / 2\rfloor} \sum_{\ell_{1}+\ell_{2}+\cdots+\ell_{n}=2 k} \prod_{j=1}^{n} \operatorname{coth}^{1-\ell_{j}} \theta_{j}}{\sum_{k=0}^{\lfloor(n-1) / 2\rfloor} \sum_{\ell_{1}+\ell_{2}+\cdots+\ell_{n}=2 k+1} \prod_{j=1}^{n} \operatorname{coth}^{1-\ell_{j}} \theta_{j}}
$$

where we have multiplied both the numerator and the denominator by the product over $j$ of $\operatorname{coth} \theta_{j}$.

When all the angles are equal, the above expressions reduce to

$$
\operatorname{coth}(n \theta)=\frac{\left.\sum_{m=0}^{n} \begin{array}{c}
m=0 \\
m
\end{array}\right) \operatorname{coth}^{n-m} \theta}{\sum_{\substack{m=0 \\
m \text { odd }}}^{n}\left(\begin{array}{c}
n \\
m
\end{array}\right) \operatorname{coth}^{n-m} \theta}=\frac{\sum_{k=0}^{\lfloor n / 2\rfloor}\left(\begin{array}{c}
n \\
2 k
\end{array}\right) \operatorname{coth}^{n-2 k} \theta}{\sum_{k=0}^{\lfloor(n-1) / 2\rfloor}\left(\begin{array}{c}
n \\
2 k+1
\end{array}\right) \operatorname{coth}^{n-2 k-1} \theta}
$$

Circular COTANGent. The circular cotangent of multiple distinct angles is given by

$$
\begin{aligned}
\cot \left(\theta_{1}+\theta_{2}+\cdots+\theta_{n}\right) & =\frac{\sum_{m \text { even }}^{n} i^{m} T_{m}^{n}\left(\cot ^{-1} \theta_{j} ; j \in N_{n}\right)}{\sum_{\substack{m=0 \\
m \text { odd }}}^{n} i^{m-1} T_{m}^{n}\left(\cot ^{-1} \theta_{j} ; j \in N_{n}\right)}, \\
\cot \left(\theta_{1}+\theta_{2}+\cdots+\theta_{n}\right) & =\frac{\sum_{k=0}^{\lfloor n / 2\rfloor}(-1)^{k} T_{2 k}^{n}\left(\cot ^{-1} \theta_{j} ; j \in N_{n}\right)}{\sum_{k=0}^{\lfloor(n-1) / 2\rfloor}(-1)^{k} T_{2 k+1}^{n}\left(\cot ^{-1} \theta_{j} ; j \in N_{n}\right)},
\end{aligned}
$$

and using definition (3.1a) for $T_{m}^{n}$, this is rewritten explicitly as

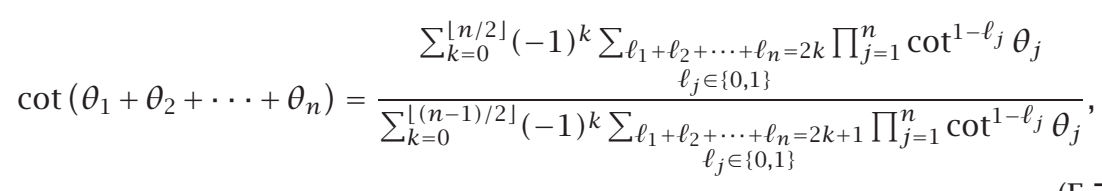

where, in the last expression, we have again multiplied the numerator and the denominator by the product over $j$ of $\cot \theta_{j}$. When all the angles are equal, the above expressions reduce to

$$
\begin{aligned}
\cot (n \theta) & =\frac{\sum_{m=0}^{n} i_{m=0}^{m}\left(\begin{array}{c}
n \\
m
\end{array}\right) \cot ^{n-m} \theta_{j}}{\sum_{\substack{m=0 \\
m \text { odd }}}^{n} i^{m-1}\left(\begin{array}{c}
n \\
m
\end{array}\right) \cot ^{n-m} \theta_{j}} \\
& =\frac{\sum_{k=0}^{\lfloor n / 2\rfloor}(-1)^{k}\left(\begin{array}{c}
n \\
2 k
\end{array}\right) \cot ^{n-2 k} \theta}{\sum_{k=0}^{\lfloor(n-1) / 2\rfloor}(-1)^{k}\left(\begin{array}{c}
n \\
2 k+1
\end{array}\right) \cot ^{n-2 k-1} \theta} .
\end{aligned}
$$


G. De Moivre-Cotes type polygonometric identities. The present paper has its origins in the relativistic problem of superposition of multiple collinear velocities [4]. Soon after terminating that work, it was realized that the results obtained are but the tip of the iceberg of a much more general problem, that of the combinatorial structure of trigonometry as presented here. Recently, Loewenthal and Robinson [15], commenting on the problem of superposition of relativistic velocities, have pointed out the existence and usefulness of a compact multiplicative De Moivre-Cotes type representation of the results. These types of identities for the polygonometric functions, actually arise in the intermediate steps of the derivation of Theorem 3.1. Even though, these compact forms correspond to a partial reversal of the proofs of the identities presented in Appendices C, D, E, and F, they are nonetheless of practical importance, especially in performing numerical computations, and are presented here for completeness. As a preliminary result, we need identities for the symmetric functions $T_{m}^{n}$ and $U_{m}^{n}$. These are derived from the closure relations.

IDENTITIES SATISFIED BY THE SYMMETRIC FUNCTIONS $T_{m}^{n}$ AND $U_{m}^{n}$. Using the scaling laws (3.3), we easily obtain

$$
\begin{aligned}
& 2 \sum_{\substack{m=0 \\
m \text { even(odd) }}}^{n} U_{m}^{n}\left(x_{j}, y_{j} ; j \in N_{n}\right) \\
& \quad=\sum_{m=0}^{n} U_{m}^{n}\left(x_{j}, y_{j} ; j \in N_{n}\right) \pm \sum_{m=0}^{n} U_{m}^{n}\left(-x_{j}, y_{j} ; j \in N_{n}\right),
\end{aligned}
$$

and using (3.2), which relate the $T_{m}^{n}$ and $U_{m}^{n}$ functions, we obtain

$$
2 \sum_{\substack{m=0 \\ m \text { even(odd) }}}^{n} T_{m}^{n}\left(x_{j} ; j \in N_{n}\right)=\sum_{m=0}^{n} T_{m}^{n}\left(x_{j} ; j \in N_{n}\right) \pm \sum_{m=0}^{n} T_{m}^{n}\left(-x_{j} ; j \in N_{n}\right)
$$

Then, making use of the closure relations (3.11) leads to

$$
2 \sum_{\substack{m=0 \\ m \text { even(odd) }}}^{n} U_{m}^{n}\left(x_{j}, y_{j} ; j \in N_{n}\right)=\prod_{j=1}^{n}\left(x_{j}+y_{j}\right) \pm \prod_{j=1}^{n}\left(-x_{j}+y_{j}\right),
$$

or equivalently,

$$
\begin{gathered}
2 \sum_{\substack{m=0 \\
\text { even(odd) }}}^{n} U_{m}^{n}\left(x_{j}, y_{j} ; j \in N_{n}\right)=\left\{\prod_{j=1}^{n}\left(1+\frac{x_{j}}{y_{j}}\right) \pm \prod_{j=1}^{n}\left(1-\frac{x_{j}}{y_{j}}\right)\right\} \prod_{j=1}^{n} y_{j}, \\
2 \sum_{\substack{m=0 \\
m \text { even(odd) }}}^{n} T_{m}^{n}\left(x_{j} ; j \in N_{n}\right)=\prod_{j=1}^{n}\left(1+x_{j}\right) \pm \prod_{j=1}^{n}\left(1-x_{j}\right) .
\end{gathered}
$$


IDENTITIES SATISFIED BY THE POLYGONOMETRIC FUNCTIONS. Applying the above results to the polygonometric identities as given in Appendices C, $\mathrm{D}, \mathrm{E}$, and $\mathrm{F}$ leads to the following hyperbolic polygonometric identities:

$$
\begin{aligned}
2 \sinh \left(\theta_{1}+\theta_{2}+\cdots+\theta_{n}\right) & =\prod_{j=1}^{n}\left(\cosh \theta_{j}+\sinh \theta_{j}\right)-\prod_{j=1}^{n}\left(\cosh \theta_{j}-\sinh \theta_{j}\right), \\
2 \cosh \left(\theta_{1}+\theta_{2}+\cdots+\theta_{n}\right) & =\prod_{j=1}^{n}\left(\cosh \theta_{j}+\sinh \theta_{j}\right)+\prod_{j=1}^{n}\left(\cosh \theta_{j}-\sinh \theta_{j}\right), \\
\tanh \left(\theta_{1}+\theta_{2}+\cdots+\theta_{n}\right) & =\frac{\prod_{j=1}^{n}\left(1+\tanh \theta_{j}\right)-\prod_{j=1}^{n}\left(1-\tanh \theta_{j}\right)}{\prod_{j=1}^{n}\left(1+\tanh \theta_{j}\right)+\prod_{j=1}^{n}\left(1-\tanh \theta_{j}\right)}
\end{aligned}
$$

and the following circular polygonometric identities:

$$
\begin{aligned}
2 i \sin \left(\theta_{1}+\theta_{2}+\cdots+\theta_{n}\right) & =\prod_{j=1}^{n}\left(\cos \theta_{j}+i \sin \theta_{j}\right)-\prod_{j=1}^{n}\left(\cos \theta_{j}-i \sin \theta_{j}\right), \quad(\mathrm{G} .7 \mathrm{a}) \\
2 \cos \left(\theta_{1}+\theta_{2}+\cdots+\theta_{n}\right) & =\prod_{j=1}^{n}\left(\cos \theta_{j}+i \sin \theta_{j}\right)+\prod_{j=1}^{n}\left(\cos \theta_{j}-i \sin \theta_{j}\right), \quad(\mathrm{G} .7 \mathrm{~b}) \\
i \tan \left(\theta_{1}+\theta_{2}+\cdots+\theta_{n}\right) & =\frac{\prod_{j=1}^{n}\left(1+i \tan \theta_{j}\right)-\prod_{j=1}^{n}\left(1-i \tan \theta_{j}\right)}{\prod_{j=1}^{n}\left(1+i \tan \theta_{j}\right)+\prod_{j=1}^{n}\left(1-i \tan \theta_{j}\right)} .
\end{aligned}
$$

In the case of equal angles, the above identities reduce, respectively, to

$$
\begin{aligned}
2 \sinh (n \theta) & =(\cosh \theta+\sinh \theta)^{n}-(\cosh \theta-\sinh \theta)^{n}, \\
2 \cosh (n \theta) & =(\cosh \theta+\sinh \theta)^{n}+(\cosh \theta-\sinh \theta)^{n}, \\
\tanh (n \theta) & =\frac{(1+\tanh \theta)^{n}-(1-\tanh \theta)^{n}}{(1+\tanh \theta)^{n}+(1-\tanh \theta)^{n}}
\end{aligned}
$$

and

$$
\begin{aligned}
& 2 i \sin (n \theta)=(\cos \theta+i \sin \theta)^{n}-(\cos \theta-i \sin \theta)^{n}, \\
& 2 \cos (n \theta)=(\cos \theta+i \sin \theta)^{n}+(\cos \theta-i \sin \theta)^{n}, \\
& i \tan (n \theta)=\frac{(1+i \tan \theta)^{n}-(1-i \tan \theta)^{n}}{(1+i \tan \theta)^{n}+(1-i \tan \theta)^{n}} .
\end{aligned}
$$

Equations (G.8) and (G.9) can also be obtained, of course, directly from the De Moivre-Cotes identity and the exponential representation of trigonometric functions. Alternatively, they can be used to provide an independent proof of the former identity. 


\section{REFERENCES}

[1] A. Aaboe, Episodes from the Early History of Mathematics, Random House, New York, 1964.

[2] M. Abramowitz and I. A. Stegun (eds.), Handbook of Mathematical Functions with Formulas, Graphs, and Mathematical Tables, National Bureau of Standards Applied Mathematics Series, vol. 55, National Bureau of Standards, Washington, D.C., 1964.

[3] W. S. Anglin and J. Lambek, The Heritage of Thales, Undergraduate Texts in Mathematics, Springer-Verlag, New York, 1995.

[4] A. F. Antippa, Relativistic law of addition of multiple collinear velocities, European J. Phys. 19 (1998), no. 5, 413-417.

[5] A. F. Antippa, J.-J. Max, and C. Chapados, Boundary effects in the hexagonal packing of rod-like molecules inside a right circular cylindrical domain. III. The case of arbitrarily oriented spherocylindrical molecules, J. Math. Chem. 30 (2001), no. 1, 31-67.

[6] H. Aslaksen, Laws of trigonometry on SU (3), Trans. Amer. Math. Soc. 317 (1990), no. 1, 127-142.

[7] C. Berge, Principles of Combinatorics, Mathematics in Science and Engineering, vol. 72, Academic Press, New York, 1971.

[8] C. V. Durell and A. Robson, Advanced Trigonometry, G. Bell \& Sons, London, 1959, first published in 1930. Reprinted 1934, 1936, 1937, 1940, 1943, 1944, 1945, 1946, 1948, 1950, 1953, 1956, 1959.

[9] N. Fleury, M. Rausch de Traubenberg, and R. M. Yamaleev, Commutative extended complex numbers and connected trigonometry, J. Math. Anal. Appl. 180 (1993), no. 2, 431-457.

[10] I. S. Gradshteyn and I. M. Ryzhik (eds.), Table of Integrals, Series, and Products, Academic Press, New York, 1980.

[11] K. Gustafson, Matrix trigonometry, Linear Algebra Appl. 217 (1995), 117-140.

[12] E. W. Hobson, A Treatise on Plane and Advanced Trigonometry, 7th ed., Dover Publications, New York, 1957, unabridged and unaltered replication of the 1928 edition. (editions 1891, 1897, 1911, 1918, 1921, 1925, 1928).

[13] R. S. Ingarden and L. Tamássy, On parabolic trigonometry in a degenerate Minkowski plane, Math. Nachr. 145 (1990), 87-95.

[14] E. Leuzinger, On the trigonometry of symmetric spaces, Comment. Math. Helv. 67 (1992), no. 2, 252-286.

[15] D. Loewenthal and E. A. Robinson, Relativistic combination of any number of collinear velocities and generalization of Einstein's formula, J. Math. Anal. Appl. 246 (2000), no. 1, 320-324.

[16] I. G. Macdonald, Symmetric Functions and Hall Polynomials, Oxford Mathematical Monographs, Oxford University Press, New York, 1998.

[17] E. Maor, e: The Story of a Number, Princeton University Press, New Jersey, 1994.

[18] _ Trigonometric Delights, Princeton University Press, New Jersey, 1998.

[19] K. S. Miller and J. B. Walsh, Advanced Trigonometry, Robert E. Krieger Publishing, New York, 1962, reprinted 1977.

[20] R. Mitra and I. I. H. Chen, The application of elliptic trigonometry to Laplace transformation and calculus, Int. J. Math. Educ. Sci. Technol 21 (1990), 161-165.

[21] B. O. Peirce, A Short Table of Integrals, Ginn \& Co., Boston, 1929.

[22] S. M. Seleby and B. Girling, Standard Mathematical Tables, The Chemical Rubber, Cleveland, 1965.

[23] D. E. Smith, History of Mathematics. Vol. I. General Survey of the History of Elementary Mathematics, Dover Publications, New York, 1958. 
[24] _ History of Mathematics. Vol. II. Special Topics of Elementary Mathematics, Dover Publications, New York, 1958.

[25] Z. W. Trzaska, On Fibonacci hyperbolic trigonometry and modified numerical triangles, Fibonacci Quart. 34 (1996), no. 2, 129-138.

[26] B. L. van der Waerden, On Greek and Hindu trigonometry, Bull. Soc. Math. Belg. Sér. A 38 (1986), 397-407.

[27] _ The motion of Venus in Greek, Egyptian and Indian texts, Centaurus 31 (1988), no. 2, 105-113.

Adel F. Antippa: Département de Physique, Université du Québec à Trois-Rivières, Trois-Rivières, Québec, Canada, G9A 5H7

E-mail address: antippa@mai 1 aps .org

URL: http://www.uqtr.ca/ antippa/ 


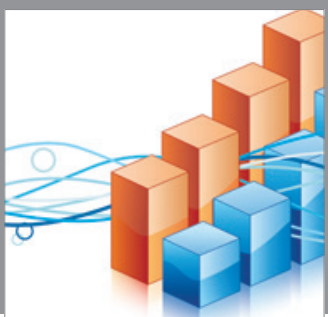

Advances in

Operations Research

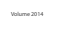

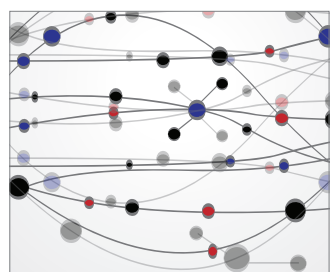

\section{The Scientific} World Journal
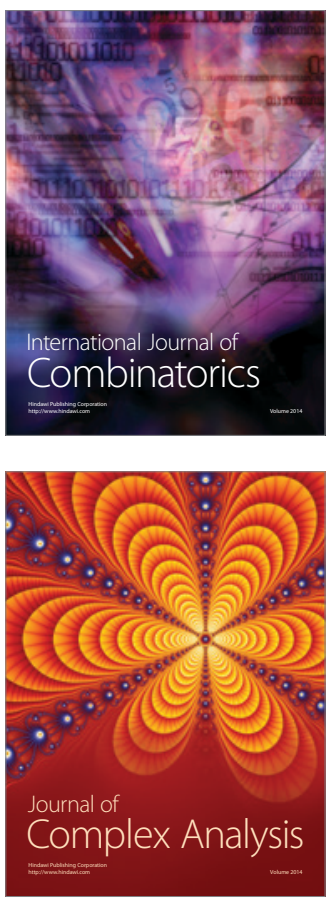

International Journal of

Mathematics and

Mathematical

Sciences
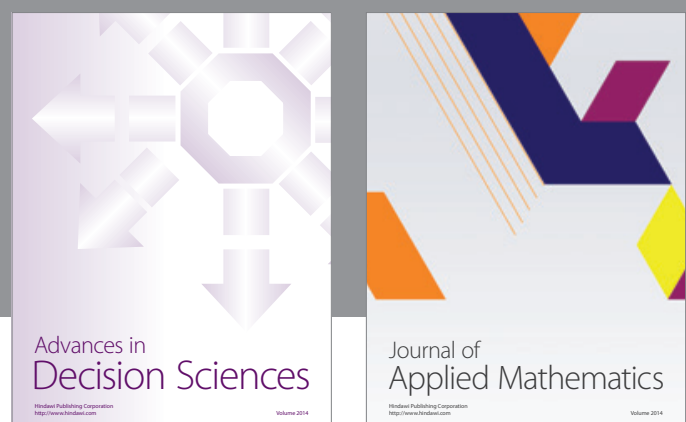

Journal of

Applied Mathematics
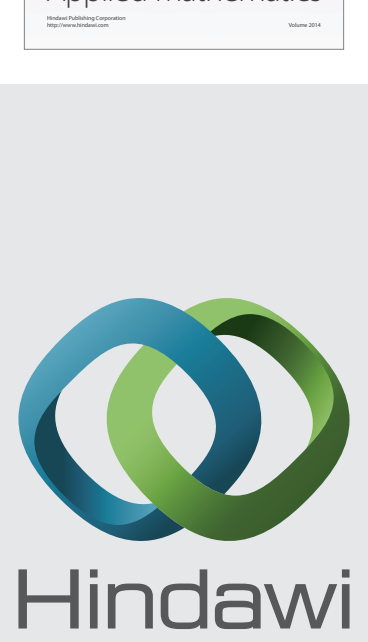

Submit your manuscripts at http://www.hindawi.com
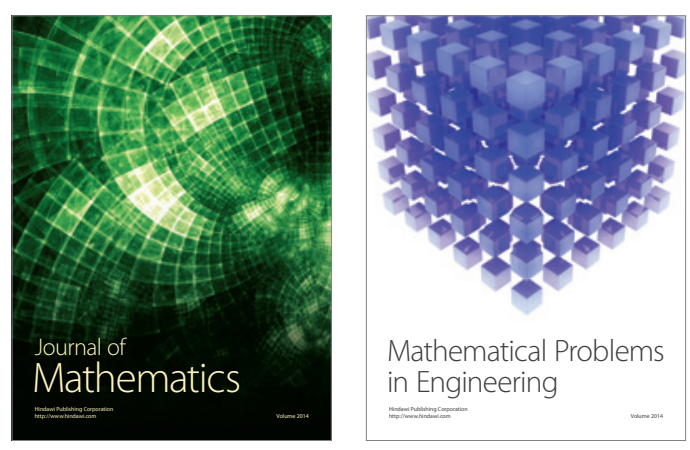

Mathematical Problems in Engineering
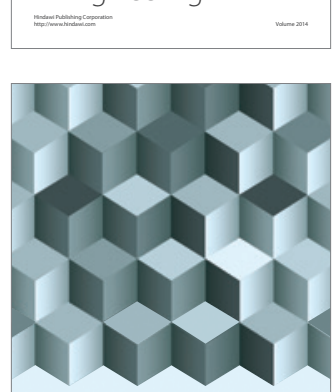

Journal of

Function Spaces
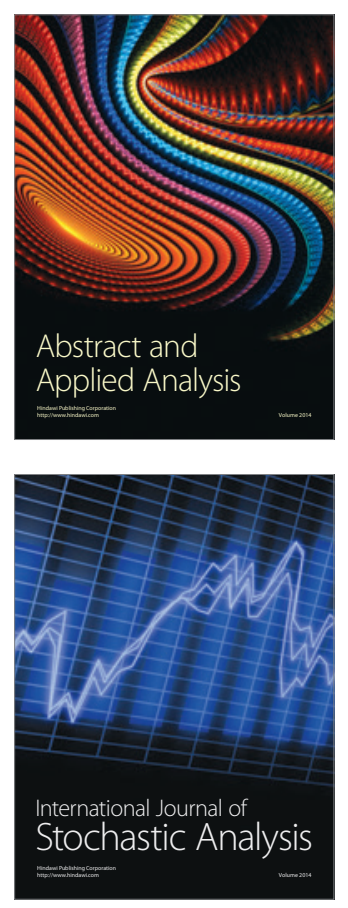

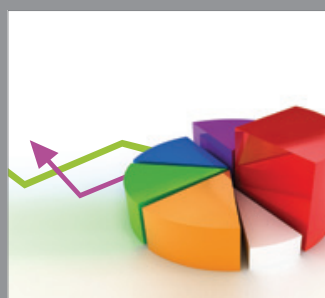

ournal of

Probability and Statistics

Promensencen
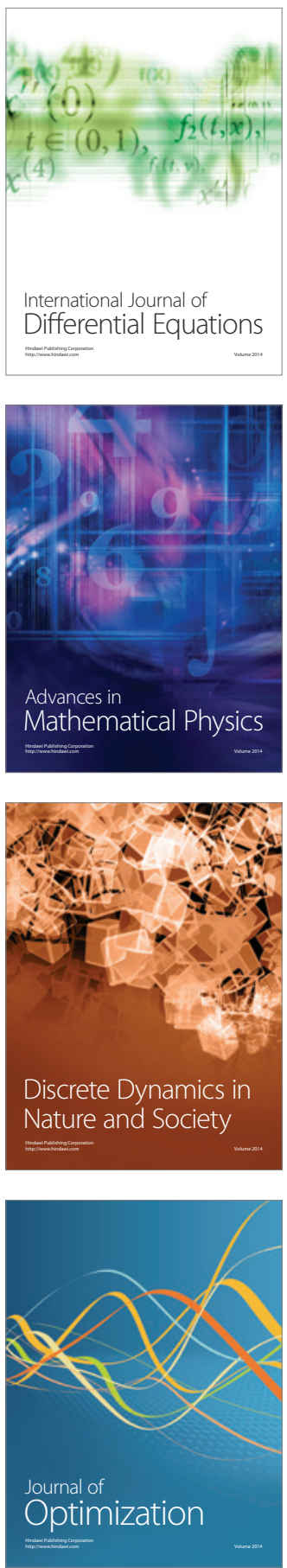